\title{
DETERMINACIÓN DE LOS ESTADIOS FENOLÓGICOS DEL FRUTO DE Vitis vinifera L. BAJO CONDICIONES DEL ALTIPLANO TROPICAL EN BOYACÁ
}

\section{DETERMINATION OF PHENOLOGICAL FRUIT STAGES OF Vitis vinifera L. UNDER TROPICAL HIGH PLATEU CONDITIONS IN BOYACÁ}

Pedro José Almanza-Merchán ${ }^{1}$ Helber Enrique Balaguera-López²

\section{RESUMEN}

En el manejo agronómico de la vid es importante el conocimiento de la fenología del fruto para establecer el momento óptimo de la vendimia y, con ello, realizar un manejo adecuado del cultivo, que conduzca a la obtención de vino de calidad. El objetivo de este trabajo fue determinar el comportamiento de los estadios fenológicos del fruto, a partir de algunos parámetros fisicoquímicos, de la variedad clonal de vid, Pinot Noir, cultivada bajo las condiciones de clima frío tropical, en la Loma de Puntalarga, Nobsa, Boyacá. Se escogieron, completamente al azar, 51 plantas. Por semana, se cosecharon tres racimos (uno por planta), se tomaron 20 frutos por racimo, recolectadas aleatoriamente, desde el día 28 después de la antesis (dda) hasta completar el desarrollo total del fruto (140dda), tiempo durante el cual, se midieron parámetros físicos y químicos del fruto. Las masas fresca y seca siguieron una curva doble sigmoide, donde la fase herbácea tuvo una duración de 63 días, el envero, 14, la maduración 42 días y la sobre maduración, 21. El pH, los sólidos solubles totales y el índice de madurez ascendieron con el desarrollo del

${ }^{1}$ Profesor Asistente. Facultad de Ciencias Agropecuarias, Universidad Pedagógica y Tecnológica de Colombia Tunja. e-mail: ppcalma@gmail.com

2 Ingeniero Agrónomo, Grupo de Investigaciones Agrícolas, Facultad de Ciencias Agropecuarias, Universidad Pedagógica y Tecnológica de Colombia Tunja. e-mail: enrique_balaguera@yahoo.com fruto, mientras que la acidez total titulable descendió. La cosecha, se debe realizar a los 126dda, puesto que el balance entre los sólidos solubles y la acidez son adecuados para la elaboración de vinos de calidad. Además, la pérdida de masa fresca y seca por efecto de la sobremaduración es mínima, lo cual, favorece tanto al productor de vino como al viticultor.

Palabras clave: Vino, uva, herbáceo, envero, maduración, sobremaduración.

\section{SUMMARY}

Knowledge of fruit phenology is important to grape growers and wine makers for proper crop handling and production of best quality wines. The objective of this study was to evaluate the behaviour of grape phenological stages, evaluating some physiochemical parameters of the clonal variety Pinot Noir, cultivated under conditions of cold tropical climate at "Loma de Puntalarga" in Nobsa, Boyacá. For this purpose 51 plants were taken at random. Weekly three bunches (one per plant) were harvested, 20 fruits per bunch were selected randomly from day 28 on after anthesis (dda) up to the fruits total development (140dda). During this time physical and chemical parameters were determined. The fresh and dry weight followed a double sigmoid curve, where the herbaceous phase lasted 63 days, the veraison 14 , maturing 42 days and over maturing 21 . The $\mathrm{pH}$, soluble solids and the maturity index increased with the fruit development, while the total acidity decreased. Harvesting should be carried out at 126dda, since at this 
moment the balance between soluble solids and total acidity is suitable for making quality wines, also at this time, the loss of fresh and dry mass by over maturing is minimal, which benefits both the wine and the grape producer.

Key words: Wine, grape, herbaceous, veraison, maturing, overmaturing.

\section{INTRODUCCIÓN}

La vid (Vitis vinifera L.), es una especie originaria de la zona templada del Asia occidental, en donde produce una cosecha al año. La vid se cultiva en los cinco continentes: Europa cuenta con 4.9000.000ha; Asia, 1.727.000ha; América con 967.000ha; África, 395.000ha y Oceanía, con 192.000ha. En América, Chile posee 191.000ha, Argentina, 219.000ha, Brasil, 78.000ha, Perú y Uruguay, 11.000ha cada uno (OIV, 2005).

En Colombia, bajo condiciones de clima frío tropical, los principales productores de vid se encuentran en el departamento de Boyacá, en altitudes entre 2200 y $2560 \mathrm{msnm}$. Allí, se ha cultivado durante los últimos 24 años, lográndose hasta dos cosechas al año, gracias a la intervención del hombre y a las condiciones agroclimáticas. A través del consorcio "Sol de oro", 64 viticultores cultivan uva con destino a la producción de vinos. En el viñedo situado en la Loma de Puntalarga, desde 1984, se cuenta con un centro experimental, donde se realiza investigación en cultivares procedentes de Geisenheim, Alemania y Borgoña, Francia (Quijano, 2004).

Jones E Davis (2000) señalan que el desarrollo de la vid ocurre como un efecto directo del clima y puede ser descrito a través de los eventos fenológicos, entendiéndose que la fenología de un cultivo es importante para determinar la capacidad de una zona o región para producir cosechas dentro del esquema de su régimen climático. El tiempo entre estadios fenológicos varía con el cultivar, con el clima y con la localización geográfica (Jones \& Davis, 2000; Tesic et al. 2002). El conocimiento de los estadios fenológicos de cultivares de vid en diferentes zonas permite el establecimiento de su capacidad adaptativa y potencial de producción (Piña \& Bautista, 2004).

El comportamiento de los estadios fenológicos del fruto de la vid está bien determinado para las variedades cultivadas en la zona templada, estos son: período herbáceo, que se caracteriza por su baya color verde y consistencia dura, engrosa y se comporta como un órgano fuente por su capacidad fotosintética y se extiende desde la formación del fruto hasta el envero; durante esta etapa, el cambio principal se manifiesta por un rápido aumento en el tamaño del fruto, consecuencia de la división celular (Reynier, 1995; Hidalgo, 1993). Luego, sigue el envero, con una duración de días a semanas y el estado, se caracteriza por una leve detención del crecimiento y por el cambio de color, que pasa del verde al color característico de la variedad; la semilla alcanza su tamaño máximo y madurez fisiológica; se inicia la síntesis de pectinas, las cuales, a través del crecimiento del fruto, son solubilizadas (Ribereau-Gayon et al. 1998; Winkler et al. 1974).

Durante el periodo de maduración, la composición de la baya cambia (Coombe E McCarthy, 2000), se observa un cambio en el color, engrosa de nuevo y se comporta como un órgano de transformación y, sobre todo, de almacenamiento. La maduración, la convierte en un almacén de reservas que, en parte preponderante y en última instancia, proviene de la sabia elaborada por los órganos verdes, regida también por las condiciones de calor y de luz que, en conjunto, condicionan la calidad de la cosecha (Hidalgo, 1993). Por último, está el periodo de sobremaduración, que se caracteriza porque el fruto pierde agua por transpiración, no genera acumulación complementaria de azúcar y la acidez disminuye, perdiéndose calidad en los vinos (Reynier, 1995; Winkler, 1948). Sin embargo, grandes vinos se hacen con sobre maduración, sin tener que ajustar la acidez.

Bajo las condiciones del trópico alto, no se ha determinado la duración y los cambios fisicoquímicos en cada periodo o estadio fenológico del fruto de uva, que permitan un manejo adecuado del cultivo y un punto óptimo de cosecha, para obtener vinos de alta calidad. La composición de la uva para vinificación y, por consiguiente, la calidad, que se puede apreciar en el vino obtenido, viene determinada por factores bióticos, abióticos y culturales. En condiciones tropicales, el factor climático es importante a la hora de decidir la programación de cosechas de vid, por tanto, el objetivo de esta investigación fue determinar los estadios fenológicos del fruto de la variedad clonal de vid, Pinot Noir, para obtener frutos de excelente valor agronómico para elaboración de vinos de calidad, cultivada bajo estas condiciones climáticas. 


\section{MATERIALES Y MÉTODOS}

La investigación, se realizó durante el año 2007 y parte del primer semestre de 2008, en el viñedo "Loma de Puntalarga”, en Nobsa, Boyacá, situado a 546’47.1” latitud Norte y $72^{\circ} 58$ '36.5” longitud Oeste, a una altura de $2618 \mathrm{msnm}$. El clima de la zona, se caracteriza por presentar una temperatura promedio anual de $16,5^{\circ} \mathrm{C}$ y precipitación bimodal media de $830 \mathrm{~mm} / \mathrm{año}$, con dos picos ubicados entre abril-mayo y octubre-noviembre. Bajo estas condiciones, se presentan temperaturas diurnas con promedio de $22^{\circ} \mathrm{C}$ y nocturnas de $8^{\circ} \mathrm{C}$, lo que origina una diferencia térmica diaria de $14^{\circ} \mathrm{C}$. Los suelos son de textura liviana, del tipo franco-arenoso y fertilidad natural baja, la insolación alcanza $476 \mathrm{cal} \cdot \mathrm{cm}^{-2} \cdot \mathrm{d}^{-1}$ en 6,5 horas diarias (Quijano, 2006).

Los frutos del genotipo, objeto de estudio, correspondieron a la variedad Pinot Noir, de la actual selección clonal de $V$. vinifera, de la cual, se elabora vino de reconocida calidad (Quijano, 2001). Las plantas tenían una edad de 24 años y estuvieron sembradas a distancia de $1,20 \mathrm{~m}$ X $2 \mathrm{~m}$, el sistema de poda es el de tipo guyot simple, con conducción en espaldera a tres alambres (Salazar E Melgarejo, 2005).

Se tomaron al azar 51 plantas; de cada una, se seleccionó un racimo homogéneo. Semanalmente, se cosecharon tres racimos, de los cuales, se apartaron 20 frutos (diez para evaluaciones físicas y diez para químicas); éstos, se escogieron aleatoriamente, desde el día 28 después de la antesis (dda) hasta completar el desarrollo total del fruto, siguiendo el procedimiento establecido por Bautista $\mathcal{E}$ Vargas (1981). Se evaluaron 60 frutos, semanalmente, para un total de 1020, durante las 17 semanas que duró la investigación.

Se obtuvo la masa fresca (MF) y la masa seca (MS), después de someter los frutos a $75^{\circ} \mathrm{C}$ durante $48 \mathrm{~h}$, el $\mathrm{pH}$, sólidos solubles totales (SST), acidez total titulable (ATT) e índice de madurez (IM), siguiendo la metodología propuesta por Parra \& Hernández (1997). La evolución de los parámetros físicos y químicos, se determinó en los laboratorios de Fisiología Vegetal de la Universidad Pedagógica y Tecnológica de Colombia, sede Tunja. Los estados fenológicos del fruto herbáceo, envero, maduración y sobre-maduración, se establecieron con base en la acumulación de MF y MS.
Los datos obtenidos, se analizaron mediante estadística descriptiva, teniendo como medida de dispersión el coeficiente de variación (CV), que fue determinado al final de cada estado y se hizo el análisis de componentes principales, con el propósito de determinar la importancia de las variables medidas en cada uno de los estadios fenológicos del fruto, teniendo como parámetro que el eje seleccionado explique más del $80 \%$ de la varianza. Se determinaron las correlaciones lineales múltiples para establecer las relaciones entre variables y para el análisis de los datos, se utilizó el programa SAS v. 8.1e (Cary, N.C).

\section{RESULTADOS Y DISCUSIÓN}

Estadio herbáceo: En la variedad Pinot Noir, el estadio fenológico se inicia con la antesis y finalizó a los 63dda (Figura 1), es decir, comprendió desde cuajado de fruto hasta envero, siendo una fase de división celular muy activa, que favorece el rápido crecimiento de la baya (Hernández, 2000; Reynier, 1995). La duración de este estado fue superior a la reportada por Hernández (2000), quien afirma que el estado herbáceo puede tener una duración entre 40 a 60 días y se caracteriza por el crecimiento de la semilla y el pericarpio, aunque hay poco desarrollo del embrión. Además, se ha encontrado que hacia las tres semanas después de antesis, la división celular del pericarpio disminuye (Mullins et al. 2000; Reynier, 1995).

El análisis mostró que con los seis componentes generados, el segundo eje explicó el 92\% de la varianza, donde las variables más representativas fueron: SST, $74 \%$ y el IM, 61\% y presentaron una correlación directa del $67 \%$ (Tabla 1). Además, la MF de las bayas, al final de este estado, llegó a $0,78 \mathrm{~g}$, mientras que la MS estuvo en 0,08g, el pH fue de 2,18, los SST de 6,63, la ATT de 4,12 y el IM fue 1,61 , todos estos parámetros presentaron poca dispersión, según el CV, excepto MS y MF, con CV de $75,6 \%$ y $77,06 \%$, respectivamente (Tabla 1 ), lo que indica que, al finalizar esta fase, los frutos no presentaron la misma acumulación de masa. Al respecto, las $M F$ y las MS aumentaron de forma significativa (Figuras 1 y 2 , respectivamente); el pH disminuyó ligeramente (Figura 3); los SST y la ATT aumentaron (Figura 4), mientras que el IM permaneció constante (Figura 5).

En el curso de este periodo, las bayas son órganos fuente en crecimiento, su respiración va en aumento 


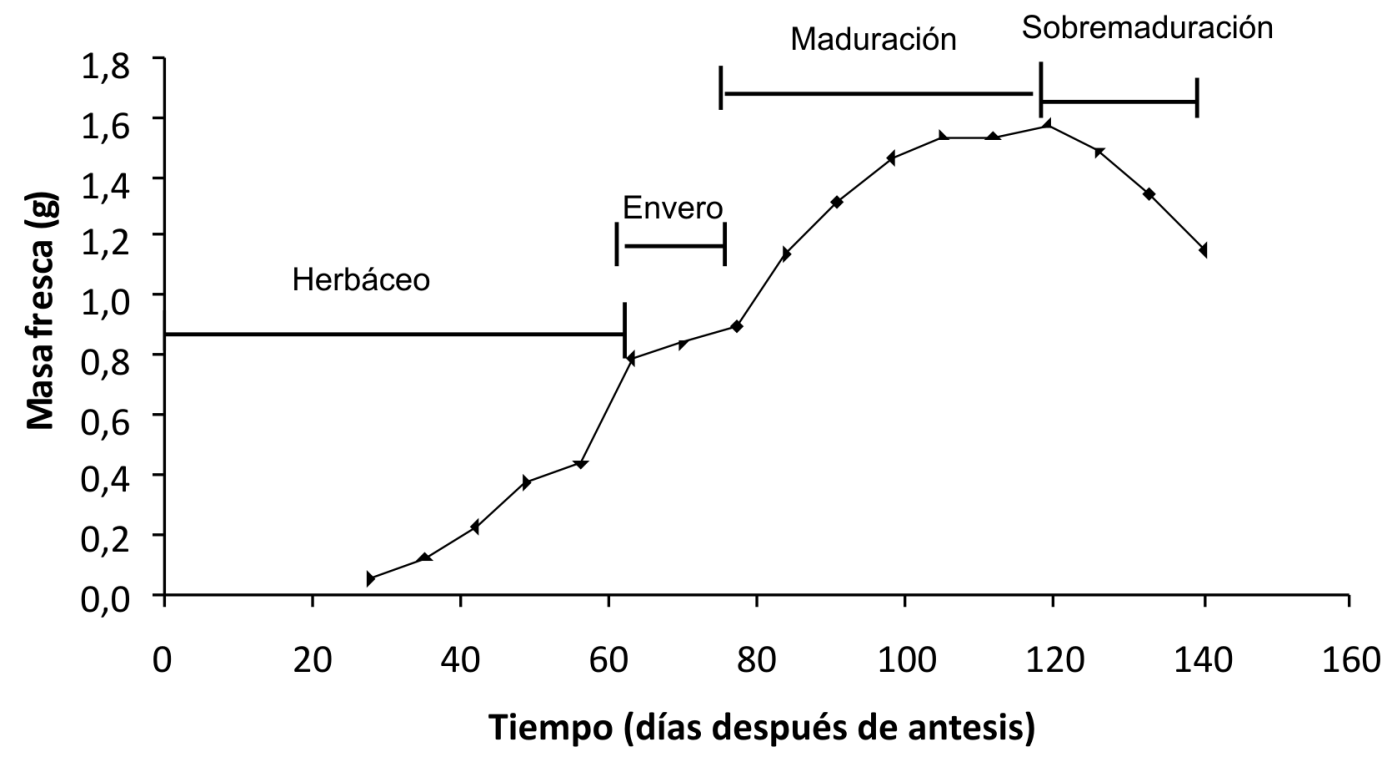

Figura 1. Comportamiento de la masa fresca en los estadios fenológicos del fruto de Vitis vinifera L., bajo condiciones de la Loma de Puntalarga, Boyacá.

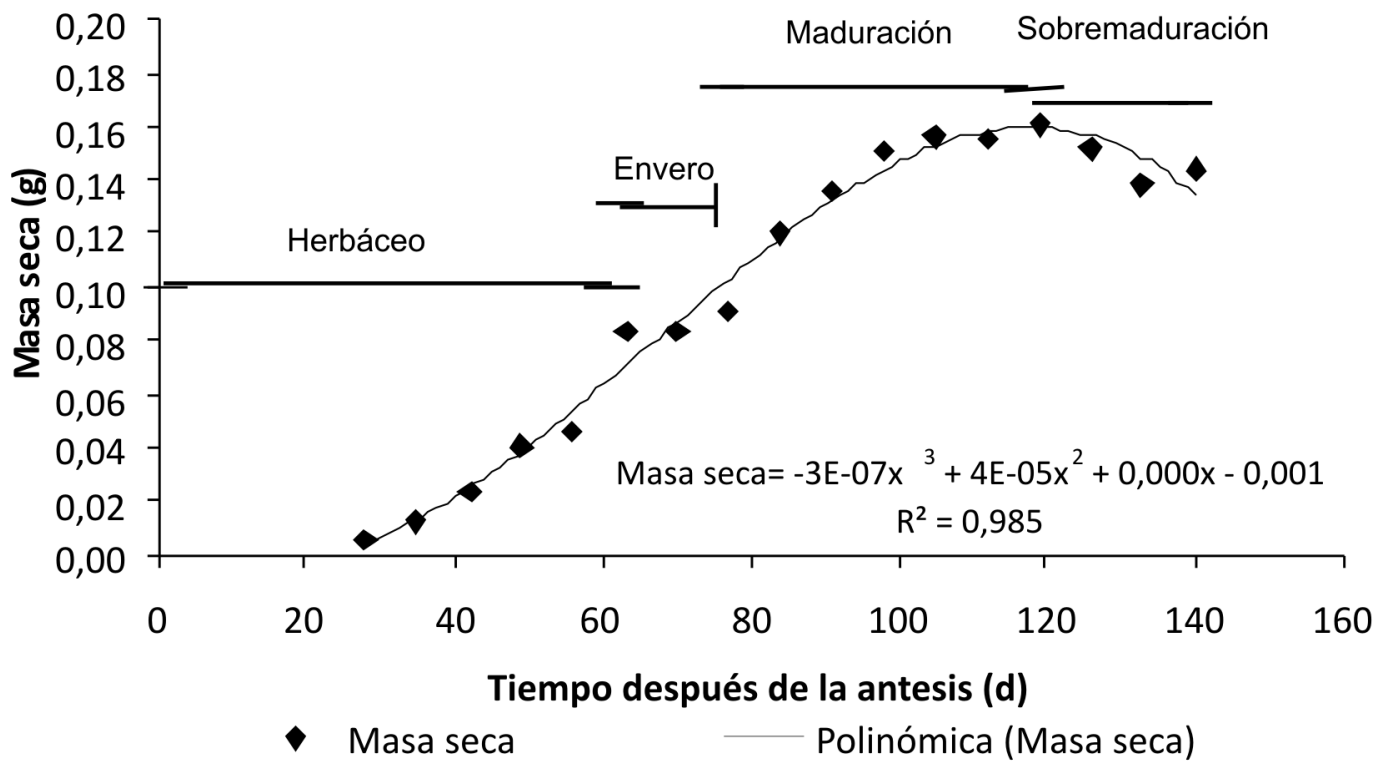

Figura 2. Comportamiento de la masa seca en los estadios fenológicos del fruto de Vitis vinifera L., bajo condiciones de la Loma de Puntalarga, Boyacá. 


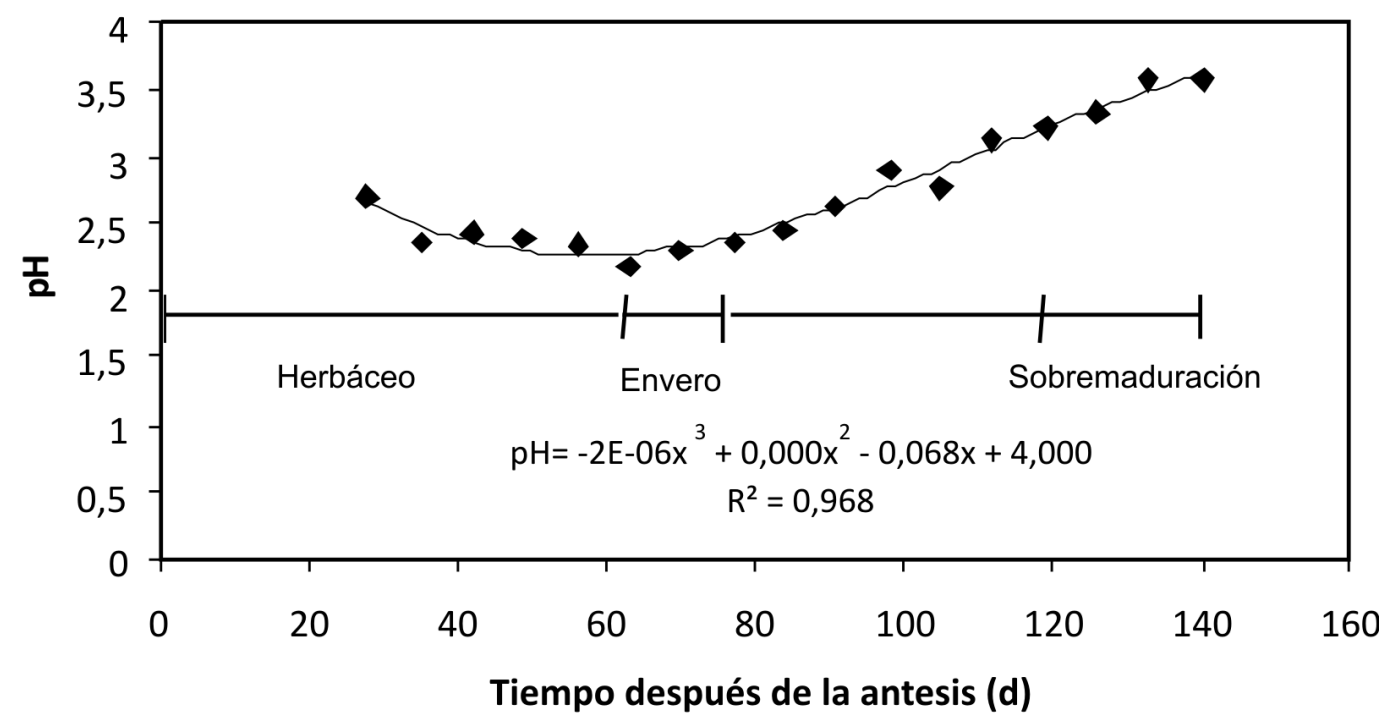

$\checkmark \mathrm{pH} \quad-$ Polinómica $(\mathrm{pH})$

Figura 3. Comportamiento del pH en los estadios fenológicos del fruto de Vitis vinifera L., bajo condiciones de la Loma de Puntalarga, Boyacá.

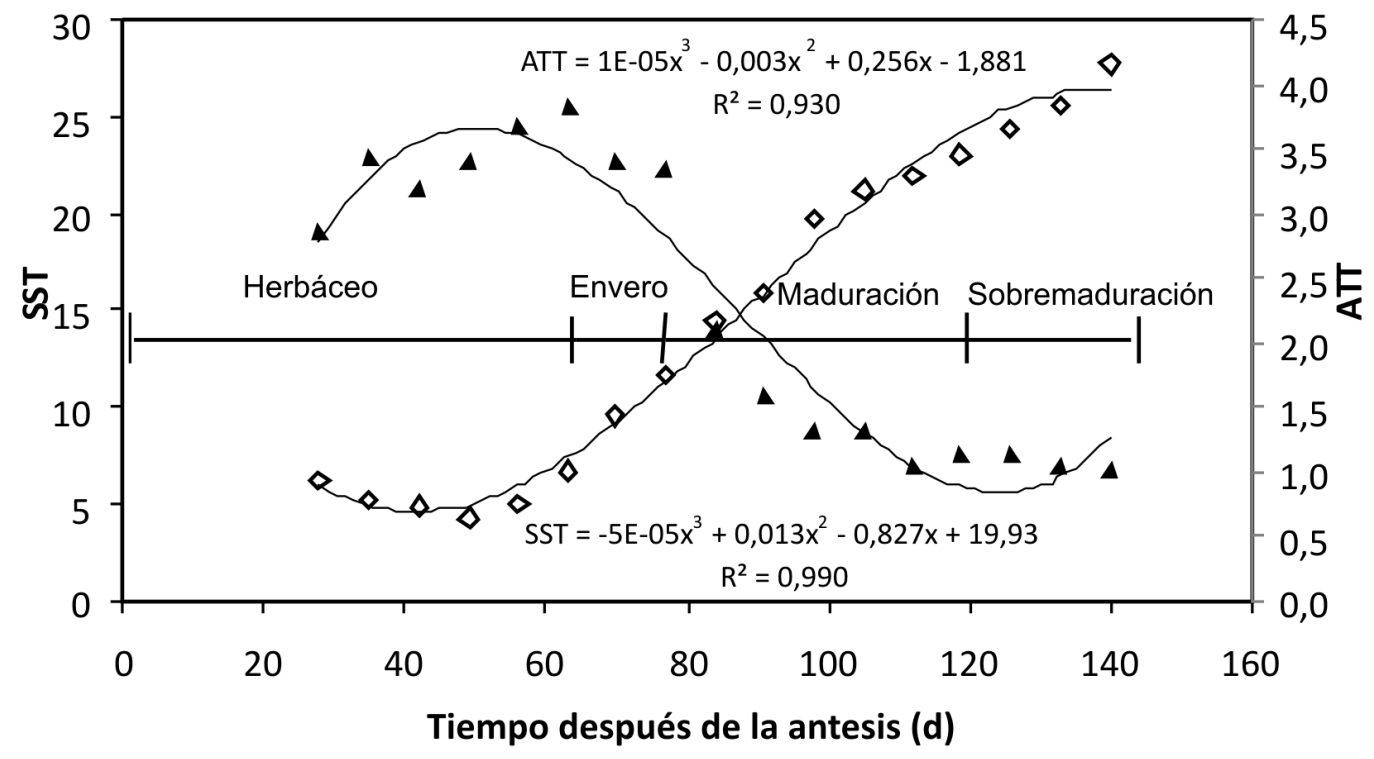

$\diamond$ SST $\quad$ ATT $\quad$ Polinómica (ATT) - Polinómica (SST)

Figura 4. Comportamiento de los SST y ATT en los estadios fenológicos del fruto de Vitis vinifera L., bajo condiciones de la Loma de Puntalarga, Boyacá. 


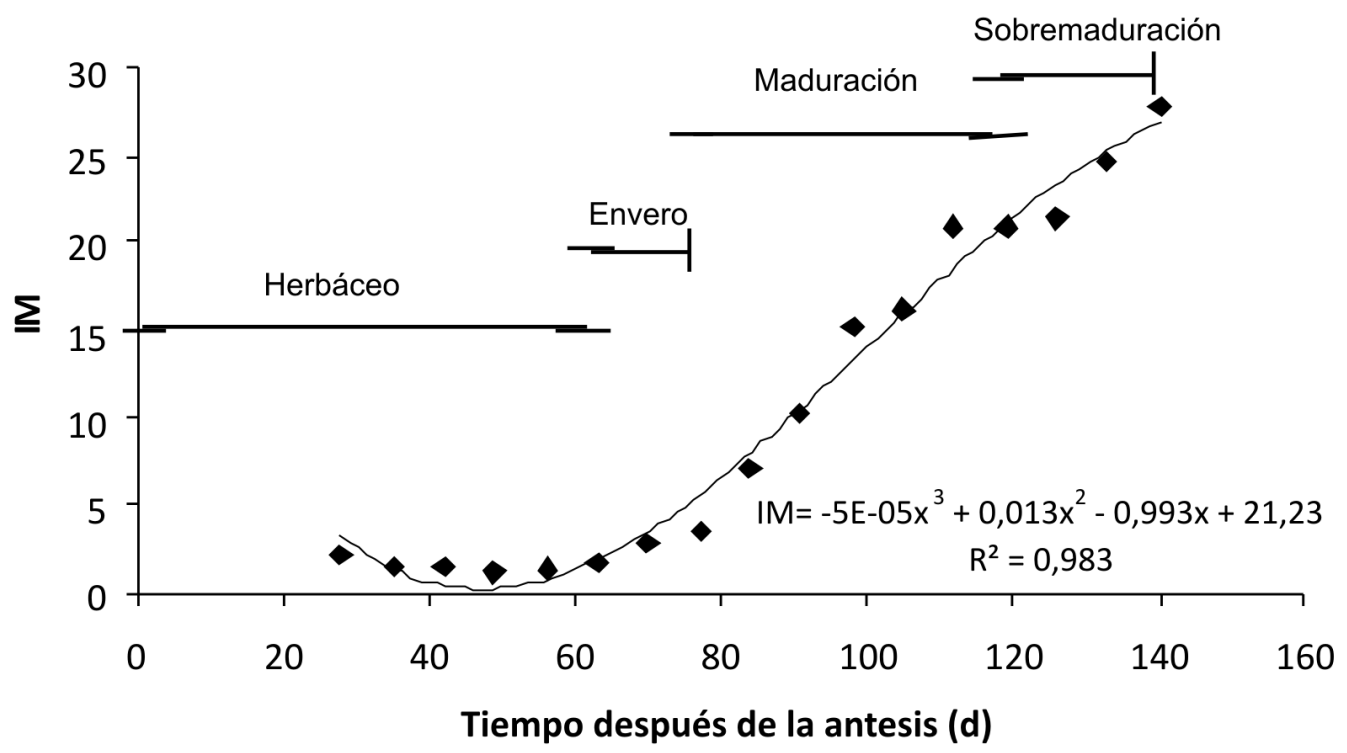

$\bullet$ IM

Polinómica (IM)

Figura 5. Comportamiento del IM en los estadios fenológicos del fruto de Vitis vinifera L., bajo condiciones de la Loma de Puntalarga, Boyacá.

hasta la mitad de este lapso, después, la fotosíntesis disminuye, de forma progresiva, hasta el final del estadio (Reynier, 1995). El comportamiento de los SST es el más representativo en esta etapa, como ya se mencionó en el análisis de componentes principales, pues los azúcares son los principales compuestos que se traslocan hacia los frutos verdes, generalmente, en forma en estado de sacarosa, que luego se hidrolizan a fructosa y glucosa. Al inicio, estos azúcares provienen de las hojas situadas a nivel de los racimos y después de aquellas de la parte media del pámpano (Reynier, 1995).

Así mismo, la biosíntesis de ácidos orgánicos, como el tartárico y el málico, es representativa y constante en el periodo herbáceo, por tanto, el contenido de SST en las bayas es bajo, mientras que el contenido de ácidos es elevado (Figura 4); esto concuerda con Reynier (1995), quien además sostiene que estos compuestos difieren según la época del año y de las variedades.

Estadio envero: El envero es el estadio más corto, puede oscilar entre siete a 40 días (Mullins et al. 2000), para todos los frutos del viñedo, pero para un solo fruto, no supera los dos días, lo que coincidió con lo encontrado en el presente estudio, ya que el envero comprendió desde los 63dda hasta los 77dda y se caracterizó porque en los 14 días de duración la MF y MS permanecieron casi constantes (Figura 1 y 2). A pesar de este fenómeno, los cambios bioquímicos fueron evidentes.

Los SST aumentaron considerablemente (Figura 4); en un principio pertenecieron a altos contenidos de glucosa y de fructosa, pero al final, predominó la concentración de fructosa (Reynier, 1995). La ATT empezó a disminuir (Figura 4), mientras que el pH aumentó levemente (Figura 3), al igual que el IM (Figura 5); el CV, en todos los casos, indicó alta homogeneidad (Tabla 1), por tanto, al finalizar el envero, los frutos presentaron similares propiedades fisicoquímicas. Esto, coincide con lo expuesto por Salazar y Melgarejo (2005), quienes afirman que en este estadio, se presenta una disminución de ácidos y acumulación rápida de azúcares y compuestos fenólicos en las bayas.

El 84\% de la varianza, se explicó con el segundo eje, el cual, demostró que la variable más representativa fue la ATT, con $81 \%$, seguida del $\mathrm{pH}$, con $47 \%$. No obstante, fueron los SST los que mostraron mayor correlación con los demás parámetros, así: IM, 98\%; MS, 72\%; pH, 65\%; MF, $48 \%$ y ATT, -23\% (Tabla 1 ).

En este estado, también se observó el cambio de color del fruto de verde a rojo oscuro, que se debe a la síntesis 
de antocianos y degradación de clorofila (Salazar E Melgarejo, 2005). Al respecto, Hernández (2000) y Winkler et al. (1974) afirman que el envero se caracteriza, porque la baya cambia de color y se pone elástica. Además, en esta fase el crecimiento del pericarpio es muy lento, lo que explica el bajo aumento de MF y MS (Figuras 1 y 2). La semilla alcanza su madurez fisiológica y el desarrollo del embrión es rápido, procesos como la respiración, la fotosíntesis y el contenido de clorofila disminuyen (Mullins et al. 2000), también el fruto inicia su recubrimiento con la pruina y los aromas herbáceos disminuyen (Hidalgo, 1993; Reynier, 1995).

Estadio maduración: Este estado tuvo una duración de 42 días, comprendidos desde los 77dda hasta los 119dda (Figura 1); esta duración es similar a lo reportado por Hernández (2000), quien afirma que el estado de maduración puede durar de 40 a 60 días en la variedad Merlot. En concordancia, Piña \& Bautista (2004) determinaron que, en condiciones tropicales, este periodo tuvo una duración de 25, 32 y 41 días para cultivares Sultanina, Perlón y Alphonse Lavallée, respectivamente. Por otro lado, se encontró que la masa fresca y seca aumentaron desde los 77dda hasta los 105dda y, luego, se estabilizaron hasta el final de este estado. Se pasó de 0,9 a $1,58 \mathrm{~g}$ y de 0,09 a $0,16 \mathrm{~g}$ para $M F$ y $M S$, respectivamente (Figura 1 y 2), aumentos muy significativos, pues según Hernández (2000), en esta etapa, el peso del fruto aumenta en un $50 \%$, aproximadamente, fenómeno que depende del suministro hídrico y del número de semillas de la variedad.

Por su parte, el $\mathrm{pH}$ se caracterizó por un marcado incremento al pasar de 2,35 a 3,22 (Figura 3); similar fue el comportamiento de los SST que alcanzó 23,07 Brix (Figura 4) y el IM incrementó hasta 20 (Figura 5), mientras que la ATT decreció pasando de 3,34 a 1,11 (Figura 4). Mullins et al. (2000) y Reynier (1995) reportan, además de los cambios mencionados para la maduración, la acumulación de minerales, formación de polifenoles, síntesis y acumulación de sustancias aromáticas, cambio en el color, ablandamiento de la baya y aumento de tamaño, el cual, está directamente relacionado con el incremento en la elongación celular y no en la división de células (Salisbury \& Ross, 1994).

Al final de la maduración, se presentó alta dispersión en las variables ATT e IM (Tabla 1). No obstante, los demás cambios fisicoquímicos medidos tienden a ser bien definidos para este cultivar. Por otro lado, una varianza del $89 \%$ fue explicada con el primer eje, según los componentes principales, este mismo análisis, pone de manifiesto la importancia de todas las variables (Tabla 1). Además, se expresa la evolución de los SST y la ATT con una correlación inversa del $90 \%$, entre estas, pero a diferencia de los demás estados, el IM presentó una correlación directa con la primera $(96 \%)$ e inversa con la segunda (-94\%). El aumento de los SST, se debe en gran parte al aumento de MF (92\%) y MS (92\%), pero determina el aumento del $\mathrm{pH}(78 \%)$ (Tabla 1).

El inicio de la maduración ocurre, normalmente, después del cese del crecimiento de los pámpanos. Durante todo el período de maduración, el metabolismo de la planta se caracteriza por una mayor fotosíntesis, de manera que la parte de azúcares degradados por respiración es más reducida que durante el período de crecimiento de los pámpanos y de las bayas verdes. El aumento de los azúcares, se presenta por el trasporte de estos a los órganos de almacenamiento, que son principalmente los racimos; los azúcares pueden provenir de la fotosíntesis, reservas presentes en tallos o por transformación del ácido málico (Reynier, 1995). Fuera de la transformación del ácido málico (gluconeogénesis), la acidez disminuye por combustión respiratoria, por la dilución, debida a la acumulación de agua y por la migración de bases procedentes de las raíces, que aumentan la alcalinidad de las cenizas (Reynier, 1995).

Estadio sobremaduración: Este es el último estadio del fruto de vid, comprendió desde el día 119 dda al 140dda (Figura 1), con una duración de 21 días. El 86\% de la varianza fue explicado con el segundo eje de acuerdo con el análisis de componentes principales. Los CV indicaron que las variables fueron muy homogéneas, lo que permite inferir que los frutos de uva Pinot Noir se comportan de manera muy similar en la sobremaduración. A su vez, la variable más importante fue el MS, con el $88 \%$ de la varianza (Tabla 1), poniendo de manifiesto que la acumulación de biomasa en el fruto de uva varía dependiendo, entre otros factores, del aporte de agua, de nutrientes, del tipo de suelo y del manejo (Hidalgo, 1993). A medida que disminuyó la MF también lo hizo la ATT, con una correlación del $67 \%$, pero aumentó el contenido de SST, IM y pH, con correlaciones de $94 \%$, $84 \%$ y $58 \%$, respectivamente (Tabla 1 ).

La MF decreció de $1,58 \mathrm{~g}$ a $1,15 \mathrm{~g}$ (Figura 1); la MS, de $0,16 \mathrm{~g}$ a $0,14 \mathrm{~g}$ (Figura 2 ) y ATT, de $1,11 \mathrm{~g}$ a $1,0 \mathrm{~g}$ 
Tabla 1. Componentes principales y correlaciones lineales para los estadios fenológicos del fruto de Vitis vinifera L.

\begin{tabular}{|c|c|c|c|c|c|c|c|c|}
\hline HERBÁCEO & MS & MF & $\mathrm{pH}$ & SST & ATT & IM & $\begin{array}{c}\mathrm{C}^{*} \\
(0,63)\end{array}$ & $\begin{array}{c}\text { C2 } \\
(0,92)\end{array}$ \\
\hline MS & 1 & 0,99 & $-0,77$ & 0,30 & 0,78 & $-0,34$ & 0,47 & 0,18 \\
\hline MF & & 1 & $-0,77$ & 0,31 & 0,79 & $-0,33$ & 0,47 & 0,19 \\
\hline $\mathrm{pH}$ & & & 1 & $-0,03$ & $-0,82$ & 0,60 & $-0,46$ & 0,08 \\
\hline SST & & & & 1 & 0,16 & 0,67 & 0,06 & 0,74 \\
\hline ATT & & & & & 1 & $-0,67$ & 0,47 & $-0,01$ \\
\hline IM & & & & & & 1 & $-0,30$ & 0,60 \\
\hline CV $(\%)$ & 75,6 & 77,06 & 6,61 & 16,52 & 13,56 & 23,31 & & \\
\hline ENVERO & MS & MF & $\mathrm{pH}$ & SST & ATT & IM & $\begin{array}{c}\text { C1 } \\
(0,62)\end{array}$ & $\begin{array}{c}\text { C2 } \\
(0,84)\end{array}$ \\
\hline MS & 1 & 0,92 & 0,63 & 0,72 & $-0,24$ & 0,46 & 0,48 & 0,00 \\
\hline MF & & 1 & 0,55 & 0,48 & $-0,02$ & 0,46 & 0,40 & 0,18 \\
\hline $\mathrm{pH}$ & & & 1 & 0,65 & 0,34 & 0,55 & 0,38 & 0,47 \\
\hline SST & & & & 1 & $-0,23$ & 0,98 & 0,47 & $-0,11$ \\
\hline ATT & & & & & 1 & $-0,39$ & $-0,10$ & 0,81 \\
\hline IM & & & & & & 1 & 0,46 & $-0,25$ \\
\hline CV $(\%)$ & 6,25 & 6,26 & 2,1 & 11,55 & 2,29 & 12,1 & & \\
\hline MADURACIÓN & MS & MF & $\mathrm{pH}$ & SST & ATT & IM & $\begin{array}{c}\text { C1 } \\
(0,89)\end{array}$ & \\
\hline MS & 1 & 0,98 & 0,75 & 0,92 & $-0,88$ & 0,86 & 0,41 & \\
\hline MF & & 1 & 0,73 & 0,92 & $-0,91$ & 0,86 & 0,41 & \\
\hline $\mathrm{pH}$ & & & 1 & 0,78 & $-0,74$ & 0,79 & 0,36 & \\
\hline SST & & & & 1 & $-0,90$ & 0,96 & 0,42 & \\
\hline ATT & & & & & 1 & $-0,94$ & $-0,41$ & \\
\hline $\mathrm{IM}$ & & & & & & 1 & 0,41 & \\
\hline CV (\%) & 10,43 & 11,85 & 10,83 & 17,07 & 26,72 & 35,85 & & \\
\hline SOBREMADURACIÓN & MS & MF & $\mathrm{pH}$ & SST & ATT & $\mathrm{IM}$ & $\begin{array}{c}\text { C1 } \\
(0,67)\end{array}$ & $\begin{array}{c}\text { C2 } \\
(0,86)\end{array}$ \\
\hline MS & 1 & $-0,12$ & $-0,19$ & $-0,10$ & 0,39 & $-0,28$ & $-0,12$ & 0,88 \\
\hline MF & & 1 & $-0,58$ & $-0,94$ & 0,67 & $-0,84$ & $-0,44$ & $-0,37$ \\
\hline $\mathrm{pH}$ & & & 1 & 0,56 & $-0,55$ & 0,58 & 0,35 & $-0,04$ \\
\hline SST & & & & 1 & $-0,78$ & 0,94 & 0,47 & 0,16 \\
\hline ATT & & & & & 1 & $-0,94$ & $-0,45$ & 0,21 \\
\hline IM & & & & & & 1 & 0,48 & $-0,04$ \\
\hline CV (\%) & 13,15 & 11,96 & 3,90 & 6,02 & 6,85 & 12,03 & & \\
\hline
\end{tabular}

${ }^{*} \mathrm{C}$ : componente principal. 
(Figura 4). El pH incrementó de 3,22 a 3,57 (Figura 3); los SST, de 23,07 a 27,77 (Figura 4) y el IM, de 20,9 a 27,77 (Figura 5). La sobremaduración llega cuando la baya alcanza su máximo tamaño, para luego iniciar la disminución en el peso (Coombe \& McCarthy, 2000), debido a que el flujo de fotoasimilados, vía floema, se interrumpe (Broussaud et al. 2001), por tanto, el fruto inicia su deshidratación, pero la concentración de SST aumenta considerablemente. Sin embargo, la síntesis de compuestos enológicos favorables (levaduras naturales, compuestos nitrogenados), se detienen y los compuestos aromáticos (linalol) y polifenoles (antocianos, flavonas, taninos) comienzan la degradación; no obstante, esta es un proceso necesario para obtener vinos tintos de calidad (Quijano, 2001).

En este momento, la baya se independiza metabólicamente, mas no funcionalmente de la planta y se hace más vulnerable a problemas fitosanitarios, como al oidio (Uncinula necator Burr.) y daños mecánicos (ataque de aves). En consecuencia, es más recomendable recolectarlo que dejarlo en la planta, siempre y cuando haya cumplido con los requerimientos fisicoquímicos del punto óptimo de cosecha, que, generalmente, es el factor más crucial para los enólogos y viticultores (Reynier, 1995).

El punto óptimo de cosecha, se puede determinar: teniendo en cuenta la evolución de los precursores glicosídicos (Jofré et al. 2006); mediante la evolución de los polifenoles (Saint-Cricq de Gaulejac et al. 1998); la madurez fenólica de la semilla (Harbertson et al. 2002); y con base en el punto de madurez tecnológica, como la relación SST/ATT (Hidalgo, 1993; Reynier, 1995), este último, es el indicador más empleado por los vitivinicultores del departamento de Boyacá.

Con base en el punto de madurez tecnológica, los frutos de Pinot Noir cultivados en condiciones agroecológicas de la Loma de Puntalarga, se deben cosechar a los 126dda, momento en el cual, se presentó un IM de 21,43, producto de una alta concentración de SST $(24,4)$, bajo contenido de ATT $(1,14)$ y $\mathrm{pH}$ de $3,33, \mathrm{MF}=1,49 \mathrm{~g}$ y $M \mathrm{~S}=0,152 \mathrm{~g}$, estas masas son considerables, al tener en cuenta que los frutos inician su pérdida de peso por procesos de respiración y de transpiración, propios de la sobremaduración. Por tanto, en este punto de cosecha, se logra obtener frutos con características químicas adecuadas, para la elaboración de vinos de calidad y con un rendimiento representativo, que favorece al productor de uva.

\section{CONCLUSIONES}

En el cultivar de vid Pinot Noir, se observaron claramente los cuatro estadios fenológicos del fruto.

En el estado herbáceo, el cambio en los sólidos solubles fue el evento más determinante.

En el estado de envero predominó la variación de la acidez total titulable.

En la maduración, la concentración de SST fue el cambio más importante y, en el estadio de sobremaduración, el factor más representativo fue la masa seca.

El punto óptimo de cosecha de uva para vino cultivar Pinot Noir cultivados en condiciones agroecológicas de la Loma de Puntalarga es a los 126dda.

\section{AGRADECIMIENTOS}

Los autores agradecen al Doctor Marco Antonio Quijano Rico, investigador y vitivinicultor, propietario del Viñedo y de la cava Loma de Puntalarga, por su apoyo en la realización del presente trabajo. Este estudio, se desarrolló con el apoyo de la Dirección de Investigaciones (DIN) de la Universidad Pedagógica y Tecnológica de Colombia, en el marco del proyecto de capital semilla convocatoria 012 de 2007, "Crecimiento y desarrollo del fruto de Vitis vinifera L. bajo condiciones de altiplano tropical", SGI 447.

\section{BIBLIOGRAFÍA}

BAUTISTA, D.; VARGAS, G. 1981. Estudio del ciclo y determinación de los requerimientos heliotérmicos de algunas variedades de vid en condiciones tropicales. Agr. Tropical (Venezuela). 31(1-6):11-23.

BROUSSAUD, F.; CHEYNIER, V.; NOBLE, A.C. 2001. Bitterness and astringency of grape and wine polyphenoles. Australian J. Grape Wine Res. 7:33-39.

COOMBE, B.; McCARTHY, M. 2000. Dynamics of grape berry growth and physiology of ripening. Australian J. Grape Wine Res. 6:131-135.

HARBERTSON, J.; KENNEDY, J.; ADAMS, D. 2002. Tannins in skins and seeds of Cabernet Sauvignon, 
Syrah and Pinot Noir berries during Ripening. Am. J. Enology Viticulture 53(1):54-59.

HERNÁNDEZ, A. 2000. Introducción al vino de Chile. Colección en agricultura de la Facultad de Agronomía e Ingeniería forestal. Pontifica Universidad Católica de Chile. 101p.

HIDALGO, L. 1993. Tratado de viticultura general. Mundi prensa, Madrid. 983p.

JOFRÉ, V.; FANZONE, M.; ASSOF, M.; RIVERA, M. 2006. Evolución de los precursores de aromas durante la maduración de uvas Cabernet Sauvignon (Vitis vinífera L.) de Agrelo y Tupungato (Mendoza, Argentina) y efecto de la maceración previa en frío. Enología. Edición internacional. 12(2):28-35.

JONES, G.R.; DAVIS, E. 2000. Climate influences on grapevine phenology, grape composition, and wine production and quality for Bordeaux, France. Am. J. Enol. Vitic. 51(3):249-261.

MULLINS, M.; BOUQUET, A.; WILLIAMS, L. 2000. Biology of the Grapevine. Cambridge, University Press. 239p.

ORGANIZACIÓN INTERNACIONAL DE LA VIÑA Y EL VINO (OIV). 2005. Situación del sector vitivinícola mundial en 2005. Disponible desde Internet en: http://news.reseau-concept.net/images/oiv_es/ client/Commentaire_Statistiques_2005_ES.pdf. (con acceso 03/08/08).

PARRA C., A.; HERNÁNDEZ, J.E. 1997. Fisiología, postcosecha de frutas y hortalizas. Bogotá. Universidad Nacional de Colombia. Facultad de Ingeniería. 64p.

PIÑA, S.; BAUTISTA, D. 2004. Ciclo fenológico de cultivares de Vid (Vitis vinifera L.) para mesa en condiciones tropicales. Bioagro. 16(1):9-16.

QUIJANO, M. 2006. Investigación e innovación. Promoción y defensa del "terroir" regional. Cultura científica, Tunja, Fundación Universitaria Juan de Castellanos. 4:35-41.
QUIJANO, M. 2004. Ecología de una conexión solar. De la adoración del sol al desarrollo vitivinícola regional. Cultura Científica. 2:5-9.

QUIJANO, M. 2001. Los vinos del valle del sol. Cultura Científica. 1:5-11.

REYNIER, 1995. Manual de viticultura. Ed. Mundi prensa. $5^{\mathrm{a}}$ ed. Madrid. 407p.

RIBEREAU-GAYON, P.; DONECHE, B.; DUBORDIEU, D.; LONVAUD, A. 1998. Traite d'oenologie. Microbiologie du vin: Vinifications. Dunod, Paris, Francia. 185p.

SAINT-CRICQDE GAULEJAC, N.; VIVAS, N.; GLORIES, Y. 1998. Maturation phenólique des raisins rouques. Relation avec la qualité des vins. Comparaison des cépages Merlot et Tempranillo. Progres Agricole et Viticole. 115:306-318.

SALAZAR, D.M.; MELGAREJO, P. 2005. Viticultura. Técnicas del cultivo de la vid, calidad de la uva y atributos de los vinos. Ed. Mundi-prensa, Madrid. 325p.

SALISBURY, F.B.; ROSS C.W. 1994. Fisiología vegetal. Grupo Editorial Iberoamérica S.A., México. 759p.

TESIC, T.; WOOLLEY, D.J.; HEWETT, E.W.; MARTIN, D.J. 2002. Environmental effects on cv. Cabernet Sauvignon (Vitis vinifera L.) grown in Hawke's Bay, New Zealand. I.. Phenology and characterization of viticultural environments. Australian J. Grape Wine Res. 8(1):15-26.

WINKLER, A.; COOK, J.; KLIEWER, N; LIDER, A. 1974. General viticulture. Univ. Cal. Press. Berkeley. 710p.

WINKLER, A.J. 1948. Maturity tests for table grapes the relation of heat summation to time. HortScience. 51:295-298.

Recibido: Agosto 4 de 2008

Aceptado: Marzo 16 de 2009 